\title{
CASE REPORT: The impact of Plasma Exeresis after fillers complication on the lips
}

\author{
PROF. T SIOUMAS, G. SOTIRIOS MD \\ Ophthalmologist - Surgeon in Ophthalmology - Acupuncturist \\ Mc Aesthetic Medicine, \\ Prof. at the University of Camerino - Torino, Italy \\ Prof. at the University of Tor Vergata - Rome, Italy \\ President of S.A.M.N.A.S. Society Aesthetic Medicine Non-Ablative Surgery \\ Vice President of the World Academy of Chinese and Complementary Medicine \\ Representative of European Medical Association (E.M.A.) in Greece \\ Vice President of Master in Aesthetic Medicine of University of Camerino-Italy in Athens \\ President and Member of the organizing Committee of the 1st and the 2nd International aesthetic Medicine \& Soft Surgery Conferences \& \\ Workshops, Athens Greece. Author of Manual of Aesthetic Medicine and Manual of Soft Surgery \\ www.ofthalmoplastiki.gr, www.tsioumasclinic.gr, www.softsurgery.gr, www.samnas.gr
}

Received: June 01, 2020; Accepted: June 22, 2020; Published: July 16, 2020

*Corresponding author: PPROF. Tsioumas G. Sotirios MD, Ophthalmologist - Surgeon in Ophthalmology - Acupuncturist, Mc Aesthetic Medicine, Prof. at the University of Camerino - Torino, Italy, Tel: +30 2108825132; E-mail: s_tsioumas@hotmail.com

\begin{abstract}
The purpose of this article is to inform the medical audience about the treatment of difficult cases by implementing the Plasma Exeresis method. With the particular device of Plasma Exeresis the fourth state of matters, Plasma, is produced. In brief, Plasma Exeresis is a certified cordless micro-surgical hand-operated device. According to the histology results, Plasma Exeresis sublimates the epidermis without passing the membrane basal, shrinking the elastic fibers of the dermis, and produces collagen type III without the unwanted skin lesion. In particular, by using Plasma Exeresis with the above mechanism we are able to rectify the fibrosis and granules (and other medical lesions e.g xanthelasmas, hemangioma, non-invasive blepharoplasty, papilloma, fibroma, benign tumors, etc.). Furthermore, it accelerates the damage repair, the wrinkles softening and the skin fibers regeneration.
\end{abstract}

The case study discussed below can be characterized for its hard and challenging nature, bearing in mind that the damage was extensive. The patient was a woman, 55 years old, who had undergone an unsuccessful treatment in the past. The case takes place in Greece. The culprit was the application of lip fillers consisted of an unknown material. The adverse effects (granules) (See pictures below) were combined with depressive disorder which lasted for approximately 15 years. The granules were considered by many doctors (even plastic surgeons) insurmountable, however, the Plasma Exeresis method was eventually highly effective. Consequently, the Plasma Exeresis can be considered as an innovative method which can heal and restore problematic cases.

Keywords: Plasma Exeresis: Fillers; Complication; Lips; Side Effects; Granules

Hypothesis 1:Plasma Exeresis can cure difficult cases

\section{Introduction}

Some of the aesthetic applications are followed by medical practices; the ones which are executed by a specialized doctor. Apart from the required scientific adequacy and professional liability, a doctor should always take into account the psychosomatic wellbeing of patients. It is a fact that various products and materials are available to the public without having the proper certifications by the issued authorities to guarantee that they are safe and effective. Another worrisome phenomenon is that many times medical treatments are executed by nondoctors or doctors who are not qualified in Cosmetic Medicine or Non-invasive Surgery and patients are not able to acknowledge that.

\section{Case study}

It will be described a case of a woman, 55 years old, who shared a story she had been living for the last 15 years; when she decided to undergo her first cosmetic treatment in a plastic doctor on the lips. She experienced depressive mood and social isolation caused mainly by her as well as due to the people's reaction when they encountered a strange sight of her face. Not to mention that she did not have a normal social and sexual life, because of the psychological impact which led to the mental disorder of depression. She outlined that "It was unfeasible to establish a connection with other people, especially when you consider your appearance repulsive".

The unsuccessful treatment concerned the application of lips filler, however, there is no indication regarding its type. Chances 
are the filler contained a prohibitive material which caused mainly by her as well as due to the people's reaction when they encountered a strange sight of her face. Not to mention that she did not have a normal social and sexual life, because of the psychological impact which led to the mental disorder of depression. She outlined that "It was unfeasible to establish a connection with other people, especially when you consider your appearance repulsive".

The unsuccessful treatment concerned the application of lips filler, however, there is no indication regarding its type. Chances are the filler contained a prohibitive material which caused an unexpected effect in the lips' area. In the upcoming period, she was confronted with purulent inflammation, granules, varicose veins, so antibiotic injections, cortisone, and others were advised as time went by. She experienced an unfavorable reality followed by long-term effects of psychological damage. She addressed to a few medical specialists (including plastic surgeons) not only in Greece but also from abroad without success. All the doctors stated that there is no way to rectify the issue. The patient visited the Tsioumas Clinic since she was interested in Noninvasive eyelids surgery. Then she was advised to start a series of Plasma Exeresis applications in the granulated zone. The patient entrusted her situation to Prof. Sotiris Tsioumas. [Figure: 1]

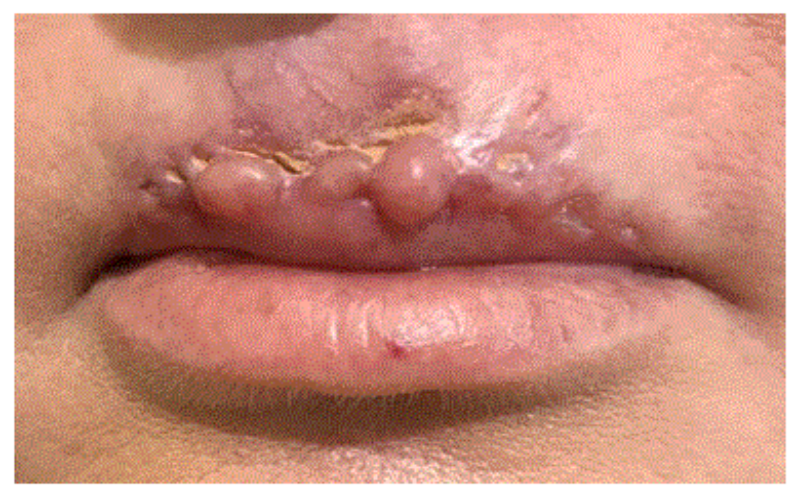

Figure 1:

\section{Methodology}

The taken action concerned the sublimation of the lesion. The Plasma Exeresis method does not pass the membrane basal, however, it aims to shrink the elastic fibers. At the first sight, the goal was to observe the granules' reaction. They had a characteristic firm and rigid form. Therefore, the procedure focused initially on the smaller side of the lesion by sublimating them using the Plasma Exeresis device. Then, crusts were developed and after seven days they left. The rehabilitation was highly successful. Consequently, we followed the same procedure since the course of actions allowed us to continue with the rest bigger granular points (in the middle lip). The whole treatment took three to four weeks and after three months a repeat session was arranged. During that period there was any unexpected deterioration.[Figure:2]

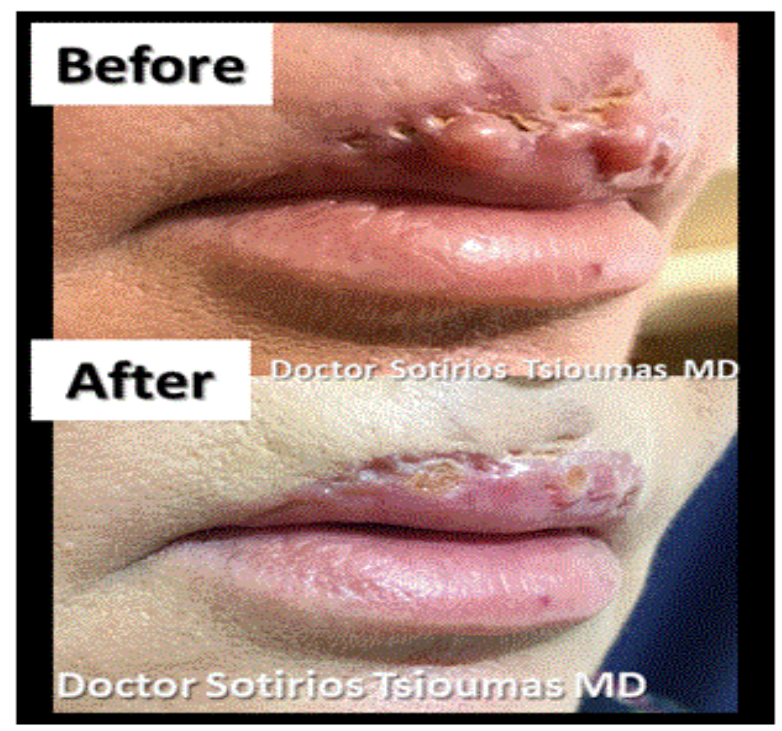

Figure 2:

\section{Results}

The patient has not reported any side effects until now. Therefore, for approximately two years she is been completely depression-free. Moreover, her appearance has been improved at a satisfactory level. The granular properties were treated and now they do not exist. The process had a remarkable positive outcome only by utilizing the Plasma Exeresis device. [Figure: 3] [Figure: 4]

\section{Conclusion}

The result was positive after a step-by-step procedure. Therefore, our hypothesis regarding the effectiveness of Plasma Exeresis can be positively supported based on the extremely difficult case. The patient disengaged herself from the effects of the past cosmetic treatment and now she can participate in common life situations. Precisely, she is now having sexual life, job, friends and she smiles again. With the Plasma Exeresis device and our accumulated substantial experience, it is possible to deal with very demanding and complicated cases like the aforementioned one. It provides functions that offer a naturallooking effect that satisfies the patient's needs. 


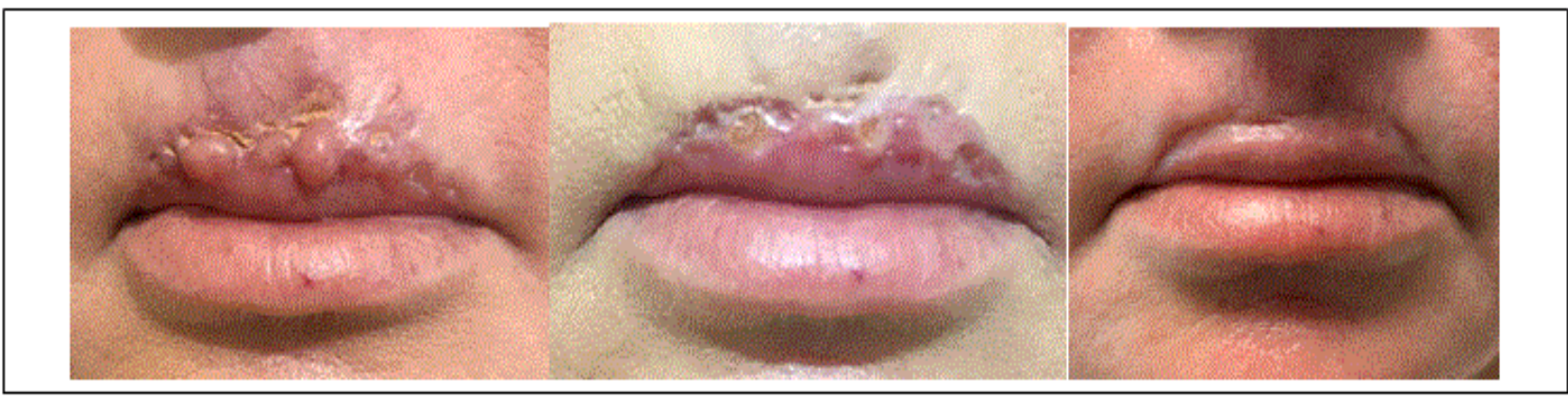

Figure 3:

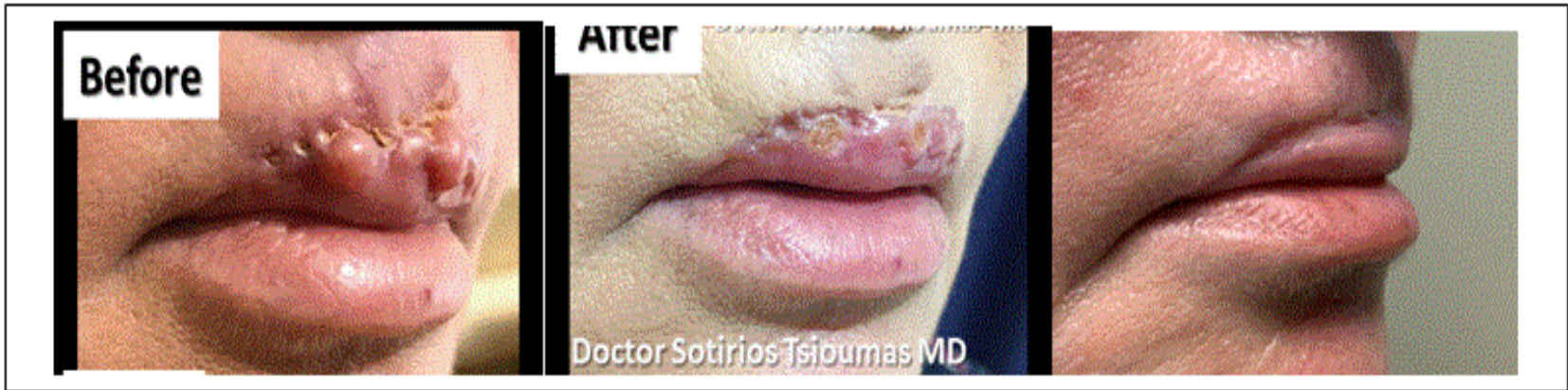

Figure 4:

\section{References}

1. Sotiris TG, Georgiadou I, Ioannis N. Noninvasive Upper Blepharoplasty in Relation to Surgical Blepharoplasty. Pinnacle Medicine \& Medical Sciences, 2014:1: 436-440.

2. Sotiris TG, Georgiadou I, Ioannis N.Plexr: The Revolution In Blepharoplasty" Pinnacle Medicine \& Medical Sciences,2014: 1: 423427.

3. Sotiris TG, Dimitris V, Nikolaos G. Clinical and Histological Presentation After Plexr Application, Needle Shaping (Vibrance) And OFF" Pinnacle Medicine \& Medical Sciences,2015: 2: 522-530

4. Sotiris TG, Ioannis C, Papapostolou A. The Efficiency of Vibrance (Needle Shaping) And O.F.F (NonSurgical Dermal Ablative Treatment) On Body Reshaping" Pinnacle Medicine \& Medical Sciences,2015: 2 : 506-509

5. Geleki S, Tsioumas S, Vranou A.Plexr In Acne Treatment" Pinnacle Medicine \& Medical Sciences,2015: 2: 482- 486

6. Sotiris TG, Ioannis C, Irini Z. The Induction of Lipolysis Using the Machine Of O.F.F (Bags Under TheEyes) And the Application of Needle Shaping Technique Using the Vibrance Machine (Tear Through)" Pinnacle Medicine \& Medical Sciences,2015: 2: 445-448

7. Sotiris TG, Ioannis C, Anastasia VL. Comparison of The Efficacy of Injectable Hyaloronic Acid Versus Needle Shaping (Vibrance) And
O.F.F (OndeFlussoFrazionato) For the Aesthetic Treatment of The Face" Pinnacle Medicine \& Medical Sciences,2015:2: 441-444.

8. Georgia G, Maria S, Sotiris GT, Dimitropoulos V, Antonio S. Presentation of Old and New Histological Results After Plasma Exercises (Plexr) Application (Regeneration of The Skin Tissue with Collagen III)" Pinnacle Medicine \& Medical Sciences,2016: 3: 983-990.

9. Sotiris TG. Georgiadis N, Georgiadou I. The Gas Ionization by Plasma Technology for Noninvasive Techniques in Oculoplastic". JOJ Ophthal,2015: 2: 555-584.

10. Sotiris TG. New Treatment with Plasma Exeresis for Non- Surgical Blepharoplasty.EC Ophthalmology,2017:4: 156-159. 